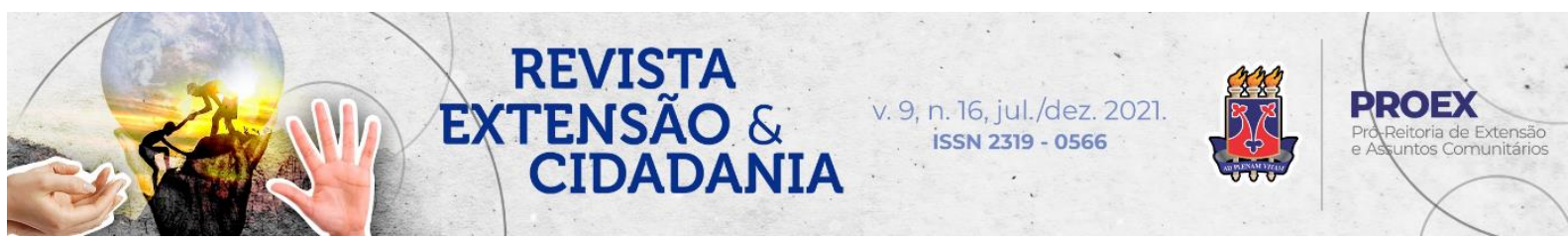

DOI: 10.22481/recuesb.v9i16.9271

\title{
OFICINAS CULINÁRIAS COMO ESTRATÉGIA PARA FORTALECIMENTO DA ECONOMIA DURANTE A PANDEMIA DA COVID-19
}

\section{KITCHEN WORKSHOPS AS A STRATEGY FOR STRENGTHENING THE ECONOMY DURING THE COVID-19 PANDEMIC}

\section{TALLERES CULINARIOS COMO ESTRATEGIA PARA FORTALECER LA ECONOMÍA DURANTE LA PANDEMIA DEL COVID-19}

\author{
Edson Douglas Silva Pontes ${ }^{1}$ \\ Mayany Carolyny Germano de Araújoº \\ Cleita Keliane do Nascimento Silva ${ }^{3}$ \\ Ana Carolina dos Santos Costa ${ }^{4}$ \\ Vanessa Bordin Viera ${ }^{5}$ \\ Raphaela Veloso Rodrigues Dantas ${ }^{6}$
}

\begin{abstract}
Resumo: A pandemia da Covid-19 impactou negativamente a economia de milhares de famílias, além das consequências na saúde humana. Diante desse contexto, foi desenvolvido o projeto de extensão COMpreender, pela Universidade Federal de Campina Grande, que uniu conhecimentos de nutrição, gastronomia, tecnologia de alimentos, marketing digital e pessoal para o fomento de renda na pandemia e fortalecimento do comércio de bebidas e alimentos por meio de receitas e informações. Dentre as ações elaboradas, destaca-se a elaboração de três oficinas culinárias realizadas de forma remota com conhecimentos práticos e teóricos sobre panificação e alimentos funcionais, com receitas previamente selecionadas e testadas. As oficinas tiveram cerca de 498 inscritos e apresentaram alta aceitação, bem como engajamento pelos participantes que tiveram a liberdade para trocar suas experiências, dúvidas e anseios diante do cenário pandêmico e como isso impactou suas vendas e rendimento familiar. Os

\footnotetext{
1 Bacharel em Nutrição, pela Universidade Federal de Campina Grande, Cuité, Paraíba, Brasil. Orcid: https://orcid.org/0000-0002-7642-9466 E-mail: edsondspontes@ gmail.com

2 Graduanda em Nutrição, Universidade Federal de Campina Grande, Cuité, Paraíba, Brasil. Orcid: https://orcid.org/0000-0002-1730-4524Ｅ-mail: mayanycarolyny@gmail.com

3 Graduanda em Nutrição, Universidade Federal de Campina Grande, Cuité, Paraíba, Brasil. Orcid: https://org/0000-0002-0571-2811 E-mail: cleitanascimento10@ gmail.com

${ }^{4}$ Doutora em Ciência e Tecnologia de Alimentos. Professora da Universidade Federal Rural de Pernambuco, Recife, Brasil. Orcid: https://org/0000-0001-5466-6463 E-mail: acarolinasc90@ gmail.com

${ }^{5}$ Doutora em Ciência e Tecnologia de Alimentos. Professora da Universidade Federal de Campina Grande, Cuité, Paraíba, Brasil. Orcid: https://orcid.org/0000-0003-4979-4510Ｅ-mail: vanessa.bordinviera@gmail.com

${ }^{6}$ Doutora em Ciências da Nutrição. Professora da Universidade Federal de Campina Grande, Cuité, Paraíba, Brasil. Orcid: https://org/0000-0002-4350-7237 E-mail: raphaelavrodrigues@yahoo.com.br
} 
relatos obtidos ao fim do projeto demonstraram grande satisfação e uma boa avaliação da metodologia adotada. Diante do que foi observado, destaca-se a importância dessas ações e da necessidade de sua continuidade para que haja um fortalecimento da economia local. Ademais, acreditamos que o projeto tem um alto valor social e que as ações desenvolvidas foram bemvistas pela população atendida.

Palavras-chave: Gastronomia. Nutrição. Segurança Alimentar e Nutricional.

Abstract: The Covid-19 pandemic negatively impacted the economy of thousands of families, in addition to the consequences for human health. In this context, The COMpreender extension project was developed by the Federal University of Campina Grande, which brought together knowledge of nutrition, gastronomy, food technology, digital and personnel marketing to boost income in the pandemic and strengthen the food and beverage trade through recipes and information. Among the actions developed, there is the development of three culinary workshops held remotely with practical and theoretical knowledge about baking and functional foods, with previously selected and tested recipes. The workshops had around 498 subscribers and showed high acceptance, as well as engagement by the participants who had the freedom to exchange their experiences, doubts and anxieties in view of the pandemic scenario and how this impacted their sales and family income. The reports obtained at the end of the project showed great satisfaction and a good evaluation of the adopted methodology. Based on what was observed, the importance of these actions and the need for their continuity are highlighted so that the local economy can be strengthened. Furthermore, we believe that the project has a high social value and that the actions developed were well regarded by the population served.

Keywords: Gastronomy. Nutrition. Food and nutrition security.

Resumen: La pandemia de Covid-19 afectó negativamente la economía de miles de familias, además de las consecuencias para la salud humana. En este contexto, el proyecto de extensión COMpreender fue desarrollado por la Universidad Federal de Campina Grande, que reunió conocimientos de nutrición, gastronomía, tecnología alimentaria, marketing digital y personal para impulsar los ingresos en la pandemia y fortalecer el comercio de alimentos y bebidas a través de recetas y información. Entre las acciones desarrolladas, se encuentra el desarrollo de tres talleres culinarios realizados de forma remota con conocimientos prácticos y teóricos sobre panadería y alimentos funcionales, con recetas previamente seleccionadas y probadas. Los talleres contaron con alrededor de 498 suscriptores y mostraron una alta aceptación, así como el compromiso de los participantes que tuvieron la libertad de intercambiar sus experiencias, dudas y ansiedades ante el escenario de la pandemia y cómo esto impactó sus ventas e ingresos familiares. Los informes obtenidos al final del proyecto mostraron una gran satisfacción y una buena evaluación de la metodología adoptada. A la luz de lo observado, se resalta la importancia de estas acciones y la necesidad de su continuidad para que se fortalezca la economía local. Además, creemos que el proyecto tiene un alto valor social y que las acciones desarrolladas fueron bien vistas por la población atendida.

Palabras clave: Gastronomía. Nutrición. Seguridad alimentaria y nutricional.

Revista Extensão \& Cidadania, v. 9, n. 16, p. 178-188, jul./dez. 2021. 


\section{Introdução}

Ao fim do ano de 2019, a cidade de Wuhan, na China, notificou uma série de casos atípicos de uma doença respiratória que possuía uma rápida disseminação. Esse vírus foi identificado como uma nova espécie de coronavírus, que ocasiona a síndrome aguda grave coronavírus-2 (SARS-CoV-2) (YUKI; FUJIOGI; KOUTSOGIANNAKI, 2020). Devido a sua rápida transmissão e a alta taxa de letalidade, a Organização Mundial de Saúde (OMS) declarou estado de emergência global ao fim do mês de janeiro de 2020, posteriormente, sob controvérsias, adotou a nomenclatura da Covid-19 para a doença ocasionada pelo novo coronavírus e, por fim, anunciando o estado de pandemia no dia 11 de março de 2020 (VELAVAN; MEYER, 2020; OPAS, 2020).

Diversas medidas de controle da doença estão sendo praticadas por vários líderes mundiais, como o distanciamento social que consiste na paralisação das atividades que proporcionem aglomerações. Dentre estas medidas, o comércio sofreu grande impacto econômico, pois trata-se de uma atividade não essencial que precisou fechar as portas durante esse período, associado ao esvaziamento dos centros urbanos houve um prejuízo na renda familiar de muitas famílias (SIPONI et al., 2020). A pandemia da Covid-19 está estritamente relacionada ao agravamento da fome no Brasil e a junção desses fenômenos configura uma enorme tragédia humanitária (FREITAS; PENA, 2020).

O empreendedorismo está associado ao inovar e apresenta alta complexidade, entretanto, empreender pode favorecer oportunidades de sucesso em negócios, desde que sejam executados da forma adequada, dentro de um contexto específico e contribuir positivamente para o desenvolvimento da economia (HATADA; MELO; CÁRNIO, 2021).

O COMpreender é um projeto de extensão da Universidade Federal de Campina Grande campus Cuité, na Paraíba, realizado por professores, estudantes e colaboradores, que visa discutir e construir junto à comunidade possibilidades para obtenção de renda com o objetivo de minimizar os impactos econômicos negativos que a Covid-19 causou em diversas famílias brasileiras.

Dessa forma, o projeto de extensão se propôs a construir, junto à comunidade, estratégias para o fortalecimento da economia local, por meio de ações voltadas ao empreendedorismo no setor de alimentos e bebidas. Dentre as ações, o presente estudo objetiva-

Revista Extensão \& Cidadania, v. 9, n. 16, p. 178-188, jul./dez. 2021. 
se a descrever um relato de experiência sobre o desenvolvimento de oficinas culinárias como ferramenta para o incentivo ao empreendedorismo e como alternativa para o fomento de renda.

\section{Metodologia}

Trata-se de um relato de experiência de um projeto de extensão, intitulado COMpreender, que foi executado por discentes do curso de bacharelado em Nutrição, pelo Centro de Educação e Saúde, da Universidade Federal de Campina Grande (CES/UFCG), na cidade de Cuité, Paraíba. Foram desenvolvidas três oficinas culinárias de alimentos, as quais continham quatro receitas, previamente testadas, desenvolvidas no Laboratório de Técnica Dietética do CES/UFCG, em que foram gravadas e, por fim, editadas para exibição aos participantes. As receitas foram ofertadas remotamente, no ano de 2020, durante a pandemia da Covid-19, conforme o Quadro 1.

\section{Quadro 1 - Receitas abordadas nas oficinas do COMpreender}

\section{OFICINA OFERTADA}

Técnicas de Panificação

Técnicas de Panificação 2.0

Alimentos Funcionais

\section{RECEITAS REALIZADAS}

Pão tradicional, pão integral, esfirra aberta e trouxinha

Pão sem glúten, empadinha, sonho, grissini de fibras

Bolo de maçã com abacaxi e nozes, bolo de banana com cacau, muffin de legumes e torta salgada low carb.

Fonte: Os autores (2021).

Participaram das oficinas os alunos de vários cursos de graduação, servidores terceirizados do CES/UFCG, comerciantes em alimentos e bebidas de diversos estados brasileiros, pessoas assistidas pelo Centro de Referência em Assistência Social (CRAS), mulheres vinculadas às comunidades carentes dos municípios de Cuité, na Paraíba, e São José do Egito, do estado de Pernambuco.

Revista Extensão \& Cidadania, v. 9, n. 16, p. 178-188, jul./dez. 2021. 
A captação das pessoas se deu por meio do Instagram, em que para se inscrever, os participantes deveriam compartilhar e marcar amigos na postagem como meio de divulgação orgânica e, posteriormente, era enviado um link para inscrição, em que continha todas as informações necessárias. O público também foi captado por grupos de Telegram e WhatsApp, os participantes que apresentaram dificuldade para realizar a inscrição, bem como não tinham acesso ao Instagram, tiveram sua inscrição facilitada pelos extensionistas para auxiliar na democratização das atividades.

Ademais, criou-se um canal no Telegram para comunicação entre os participantes e os extensionistas para tirar dúvidas remanescentes, além de fomentar um espaço para troca de experiências entre os participantes. Após a primeira oficina, foi solicitado de forma expressiva outras oficinas e, junto a isso, sugestões que foram analisadas e abordadas nas ações posteriores. Cada oficina teve duração média de 1 hora, em que 20 minutos eram destinados a parte teórica sobre técnicas de panificação e alimentos funcionais e prática consistia em 40 minutos com vídeos com o passo-a-passo das receitas elaboradas com narração e explicação simultânea do extensionista responsável.

Também, foram desenvolvidas cartilhas educativas, contendo um resumo da parte teórica, a descrição das receitas, rendimento, ingredientes e suas quantidades, modo de preparo e sugestões para venda e consumo dos produtos elaborados.

\section{Resultados e discussão}

A iniciativa contou com a participação de 498 inscritos, na faixa etária de 18 a 50 anos de idade, por meio de uma plataforma de Streaming. Foi utilizado recursos midiáticos como slides com imagens, textos informativos e vídeos para proporcionar uma compreensão dos assuntos propostos de forma simplificada. Desse modo, os indivíduos tinham a liberdade de assistir as gravações durante as primeiras 120 horas.

O uso de atividades digitais é uma das principais estratégias utilizadas por microempreendedores para reverter a crise financeira ocasionada pela pandemia, objetivando atrair e engajar os clientes com ações voltadas às divulgações em redes sociais e o marketing digital se consolidou como um dos únicos meios para manter a proximidade com as pessoas (SILVA, 2021). Desse modo, o empreendedorismo compreende uma deliberação consciente, baseada na análise de oportunidades e do reconhecimento da necessidade de encontrar lacunas

Revista Extensão \& Cidadania, v. 9, n. 16, p. 178-188, jul./dez. 2021. 
no mercado de trabalho, em que se torna imprescindível a criação de um novo negócio ou produto (RATTEN, 2020).

Para manter esse vínculo com os participantes, foram criados alguns grupos nas mídias sociais, tais como o Telegram e o WhatsApp, com o intuito de melhorar a comunicação entre eles, por meio da liberação de informações sobre as oficinas, links destinados aos posts informativos no Instagram. Também, foi empregado para sanar as possíveis dúvidas que viessem a surgir e disponibilizar os materiais didáticos referentes a cada oficina, que eram apresentadas nas gravações, a fim de facultar o ensino-aprendizado.

Inicialmente, ocorreu a apresentação da equipe, a difusão do projeto e a sua devida finalidade. Foi abordado os seguintes tópicos: "Introdução à tecnologia de panificação" com a explanação dos dados referentes à produção e ao faturamento da indústria alimentícia, no Brasil, além de exibir os diferentes tipos de pães e os seus conceitos e "Etapas no processo de panificação", que tratou dos princípios sobre o glúten, gliadina, glutenina, fermentação e forneamento. Ressaltou-se, também, as técnicas de pesagem, mistura, descanso e temperatura ideal para a produção da massa, assim como o método para o boleamento, seguida da fermentação e o forneamento.

Em suma, vale destacar que existem diversos métodos utilizados para melhorar a qualidade dos produtos, entre eles estão as técnicas citadas, que são caracterizadas por proporcionar a padronização, a fim de conferir o controle da produção, evitar desperdícios e prejuízo à empresa. Na Oficina "Técnicas de Panificação", explanou-se sobre o modo de preparo de algumas receitas (pão tradicional, pão integral, esfirra aberta e trouxinha), as quais foram desenvolvidas almejando propiciar à comunidade estratégias de geração de renda financeira para o enfrentamento da crise econômica, através de preparações palatáveis e de baixo custo.

A Oficina “Técnicas de Panificação 2.0” retratou as preparações (pão sem glúten, empadinha, sonho, grissini de fibras) e os procedimentos para a confecção em que incluíam os ingredientes, temperatura ideal e o passo a passo para a elaboração de cada produto, objetivando viabilizar aos ouvintes a aptidão e a sua uniformização.

Por último, apresentou-se a Oficina “Alimentos Funcionais”, a qual foi explanada o conceito e os benefícios da ingestão desses alimentos na saúde do consumidor (Bolo de maçã com abacaxi e nozes, bolo de banana com cacau, muffin de legumes e torta salgada low carb). Além do mais, abordou-se acerca dos compostos fenólicos, carotenoides, ômega 3, fibras e probióticos,

Revista Extensão \& Cidadania, v. 9, n. 16, p. 178-188, jul./dez. 2021. 
os quais elencou-se as suas definições, funções e classes, de maneira descomplicada e objetiva, com o intuito de facilitar o entendimento dos ouvintes, dado que a maior parte não detinha acesso ao ensino superior.

Torna-se relevante elucidar sobre estes tópicos para que o consumidor possa entender como cada nutriente irá atuar na sua saúde e, também, como algumas técnicas de cocção são capazes de ativar ou não os biodisponíveis para que se possa traçar o perfil da comida e da clientela que almeja trabalhar. Também, é importante conhecer estes conceitos para ter maior embasamento teórico ao vender o que foi produzido e convencer o cliente a comprar o alimento, ressaltando que este pode ser tanto saboroso quanto nutritivo.

Ao término do curso de formação, realizou-se uma pesquisa entre os ouvintes para detectar os pontos positivos e negativos dessa atividade. Com base nisso, foi possível observar a existência de uma boa satisfação quanto aos conteúdos ministrados, sobretudo, a temática de alimentos funcionais, conforme Figura 1.

\section{Figura 1 - Posicionamento dos participantes em relação à preferência da oficina digital}

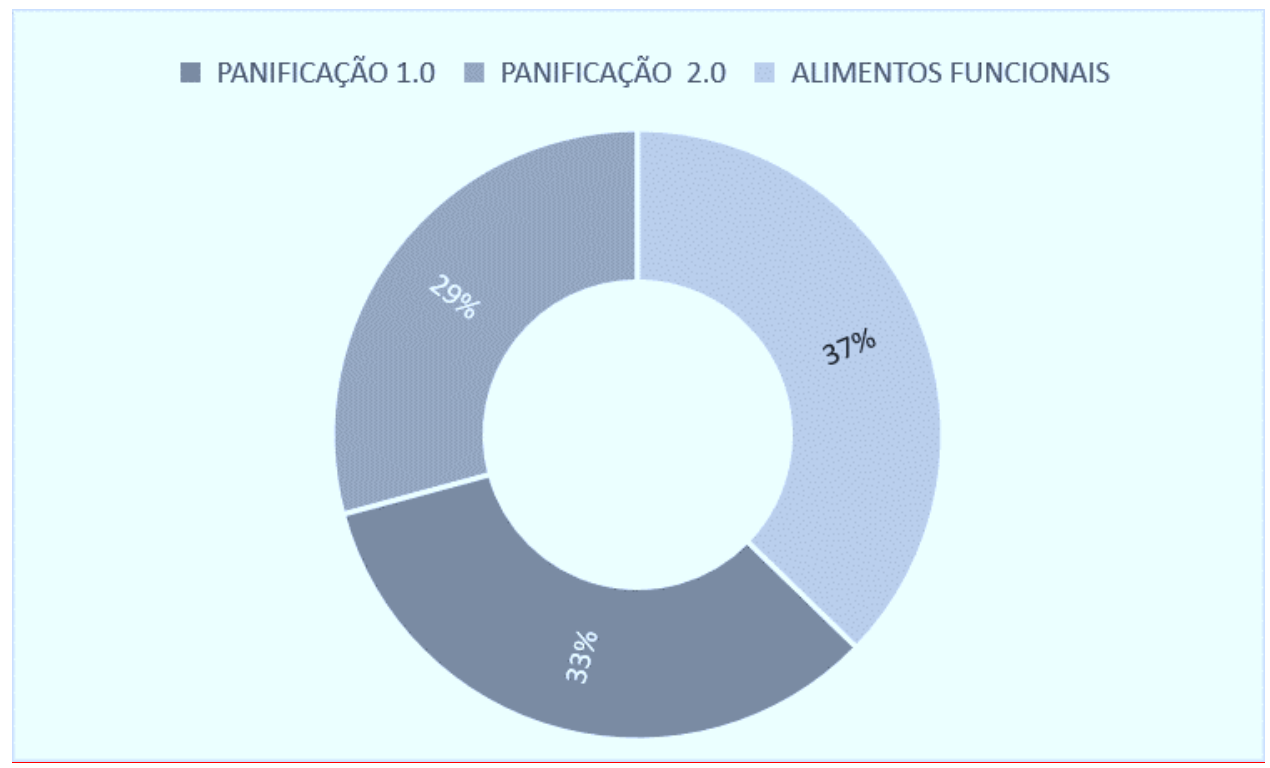

Fonte: Elaborado pelos autores (2021).

Para aprofundar esta avaliação, foi necessária uma análise da percepção dos participantes em relação às oficinas virtuais. Para tal, primordialmente, deve-se considerar a primazia e percepção do aluno quanto à idealização do curso para obtenção de um ensinamento

Revista Extensão \& Cidadania, v. 9, n. 16, p. 178-188, jul./dez. 2021. 
de maior eficácia. Por conseguinte, para expandir essa concepção, a análise da temática a ser ministrada pelo participante é designada como um instrumento útil para o sucesso desta ação (MUTHUPRASAD; AISWARYA; ADITYA; GIRISH, 2021).

Os participantes entrevistados, em sua maioria, preferiram a Oficina de Alimentos Funcionais, de acordo com Silva (2019), existe uma preocupação com uma alimentação saudável que nutra e promova a saúde, resultando no aumento pela preferência dos consumidores por alimentos ricos em nutrientes que possam fortalecer o organismo, além de prevenir e combater doenças.

Posto isto, as escolhas das temáticas aludidas foram desenvolvidas como estratégias de empreendedorismo, visando proporcionar a população um melhor enfrentamento econômico, através do compartilhamento de saberes durante o momento da capacitação, com o objetivo de ofertar uma ampliação do conhecimento, autonomia e criatividade aos internautas para que adquissem novas oportunidades no mercado de trabalho.

Outrossim, foi possível observar que as oficinas obtiveram uma aceitação satisfatória quanto a sua didática, contribuindo, assim, ao processo de aprendizagem dos inscritos, conforme Figura 2.

\section{Figura 2 - Avaliação dos participantes quanto da oficina digital ofertada remotamente}

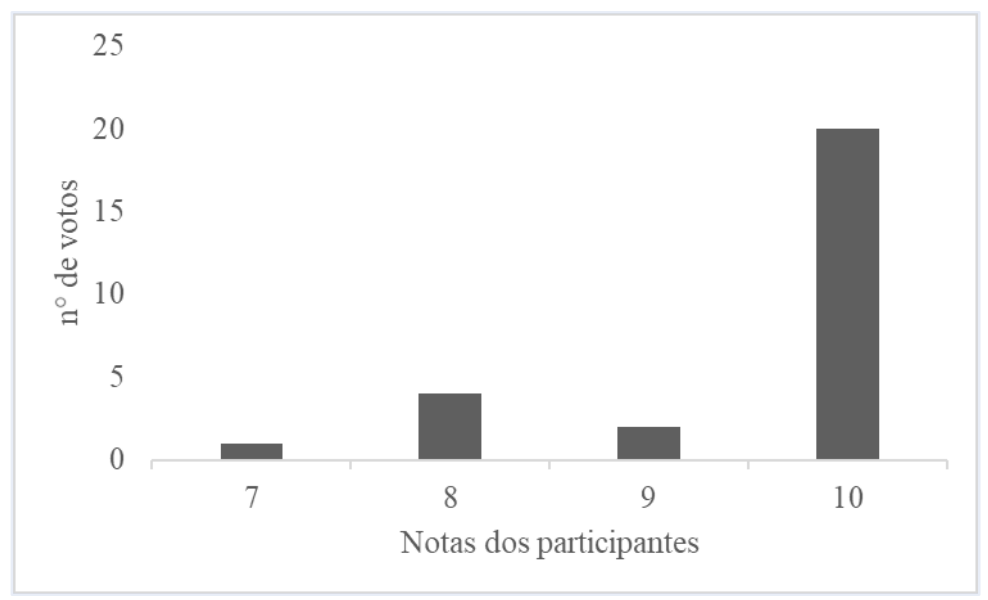

Fonte: Elaborado pelos autores (2021).

A interação do aluno com o palestrante, de acordo com Martin, Wang e Sadaf (2018), é caracterizada como uma ferramenta essencial para a facilitação do aprendizado em cursos virtuais, a exemplo disso, são os vídeos narrados em arquivos de powerpoint, o qual é

Revista Extensão \& Cidadania, v. 9, n. 16, p. 178-188, jul./dez. 2021. 
considerado o método de ensino preferido pelos estudantes (HAMPTON; PEARCE; MOSER, 2017).

Esses achados se equivalem com a metodologia deste estudo, em que os conteúdos abordados foram disponibilizados nas gravações supracitadas, além de haver o contato direto com os inscritos no canal do Telegram, com o intuito de sanar as possíveis dúvidas, e o acesso às cartilhas. Os materiais complementares, baseados em textos, são classificados como uma tática menos eficaz de aprendizagem, conforme Hampton et al. (2020), dado que os alunos têm preferência por conteúdos objetivos. Com relação à qualidade do material didático, referente à oficina digital, observou-se um grau de aprovação acentuada, como pode ser observado na Figura 3.

\section{Figura 3 - Avaliação dos participantes quanto à qualidade dos materiais didáticos}

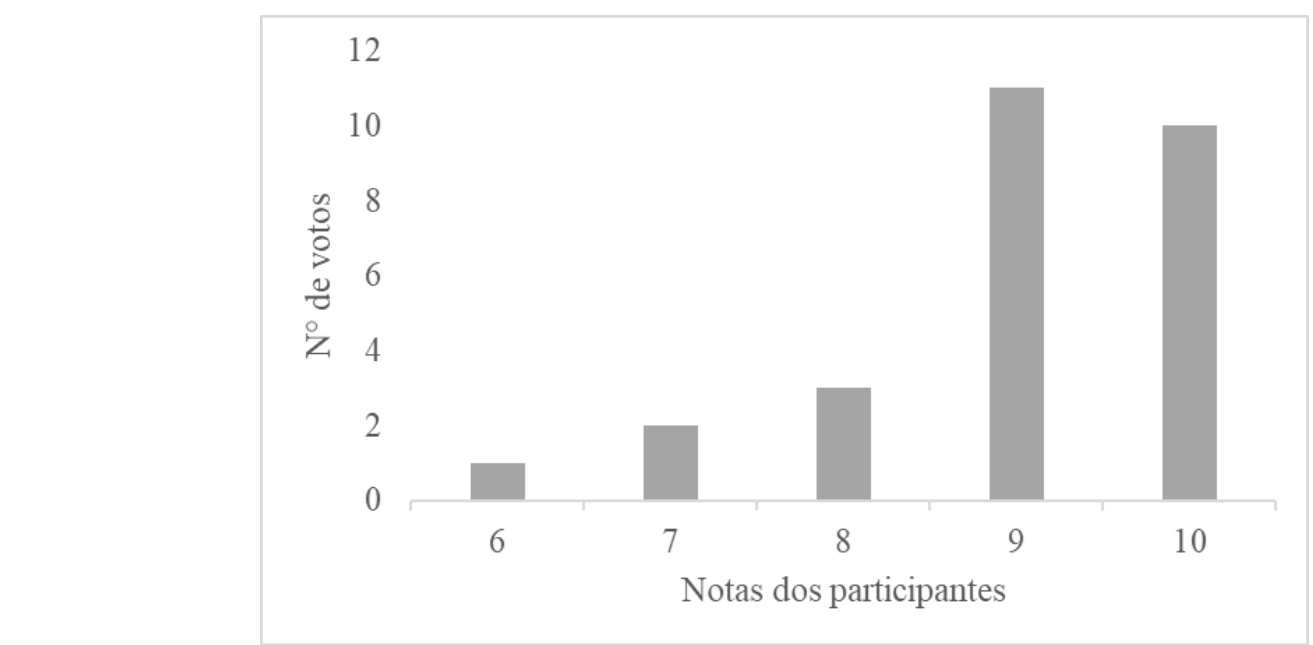

Fonte: Elaborado pelos autores (2021).

As contrariedades decorrentes da criação do conteúdo didático via tecnologia digital de informação e comunicação não são experiências novas ou inéditas, considerando-se que a modalidade de ensino à distância está presente há vários anos (DOURADO et al., 2021). Nessa perspectiva, para a criação dos materiais houve, inicialmente, um planejamento dos conteúdos, a fim de garantir um recurso interativo com características marcantes, dinâmicas e de hipertextualidade, além de serem gratuitas e de fácil acessibilidade.

A informatização do meio escolar remoto é identificada por ser algo oportuno ao aprendizado do aluno, conforme Lopes e Fürkotter (2016), devido a vasta disponibilidade de

Revista Extensão \& Cidadania, v. 9, n. 16, p. 178-188, jul./dez. 2021. 
softwares e conteúdos digitais educacionais que promovem a flexibilidade ao acesso concedido pela dinâmica da internet e, também, os benefícios que as tecnologias e materiais didáticos, quando introduzidas de maneira ordenada, podem oferecer.

\section{Conclusão}

Os resultados deste estudo indicaram que a maioria dos participantes evidenciou uma atitude positiva em relação às oficinas, principalmente, nas temáticas dos alimentos funcionais e panificação. Posto isto, o aprendizado remoto mostrou-se vantajoso, pois proporcionou flexibilidade, fortalecimento da autonomia, criatividade e estímulo para geração do incremento da renda familiar a partir dos conhecimentos construídos nas oficinas.

\section{Referências}

DOURADO, A. C.; SOUSA, Cícero J. M.; SOUTO, T. V. S. Relato de experiência de uma intervenção didática sobre a origem do universo em um contexto de ensino remoto. Instituto Federal de Pernambuco Campus Pesqueira, Curso de Licenciatura em Física. Pesqueira, 2021.

FREITAS, M. C. S.; PENA, P. G. L. Fome e pandemia da Covid-19 no Brasil. Tessituras: Revista de Antropologia e Arqueologia, v. 8, n. 1, p. 34-40, 2020.

HAMPTON, D.; CULP-ROCHE, A.; HENSLEY, A.; WILSON, J.; OTTS, J. A.; THAXTON-WIGGINS, A.; MOSER, D. K. Self-efficacy and satisfaction with teaching in online courses. Nurse educator, v. 45, n. 6, p. 302-306, 2020.

HAMPTON, D.; PEARCE, P. F.; MOSER, D. K. Preferred methods of learning for nursing students in an on-line degree program. Journal of Professional Nursing, v. 33, n. 1, p. 27 37, 2017.

HATADA, F.; MELO, C. G. L.; CÁRNIO, T. C. Empreendedorismo e intervenção estatal em tempos de pandemia. MISES: Interdisciplinary Journal of Philosophy, Law and Economics, v. 9, 2021.

LOPES, R. P.; FÜRKOTTER, M. Formação inicial de professores em tempos de TDIC: uma questão em aberto. Educação em Revista, v. 32, p. 269-296, 2016.

MARTIN, F.; WANG, C.; SADAF, A. Student perception of helpfulness of facilitation strategies that enhance instructor presence, connectedness, engagement and learning in online courses. The Internet and Higher Education, v. 37, p. 52-65, 2018.

Revista Extensão \& Cidadania, v. 9, n. 16, p. 178-188, jul./dez. 2021. 
MUTHUPRASAD, T.; AISWARYA, S.; ADITYA, K. S.; GIRISH K. Students' perception and preference for online education in India during Covid-9 pandemic. Social Sciences \& Humanities Open, v. 3, n. 1, p. 100-101, 2021.

OPAS. Organização Pan-Americana de Saúde. Histórico da pandemia da Covid-19. Disponível em: https://www.paho.org/pt/covid19/historico-da-pandemia-covid-19. Acesso em: 7 jun. 2021.

RATTEN, V. Coronavírus (Covid-19) e empreendedorismo: mudando a paisagem de vida e trabalho. Journal of Small Business \& Entrepreneurship. v. 32, ed. 5, p. 503-516, 2020.

SILVA, A. R. C. Estratégias empreendedoras implantadas pelos microempreendedores campinenses na pandemia da Covid-19. 2021. 30f. Trabalho de Conclusão de Curso (Graduação em Administração) - Universidade Estadual da Paraíba, Campina Grande, 2021.

SILVA, V. S.; ORLANDELLI, R. C. Desenvolvimento de alimentos funcionais nos últimos anos: uma revisão. Revista Uningá, v. 56, n. 2, p. 182-194, 2019.

SIPIONI, M. E.; RIQUIERI, M. R. L.; BARBOSA, J. P. M.; BISCOTTO, D. B.; SARTI, T. D.; ANDRADE, M. A. C. Máscaras cobrem o rosto, a fome desmascara o resto: Covid-19 e o enfrentamento à fome no Brasil. 2020. Disponível em:

file:///C:/Users/User/Downloads/jeaninepacheco,+Fome+e+COVID_ScieloPreprint.pdf.

Acesso em: 31 maio 2020.

VELAVAN, T. P.; MEYER, C. G. The Covid-19 epidemic. Tropical Medicine \&

International Health, [S.L.], v. 25, n. 3, p. 278-280, 16 fev. 2020.

YUKI, K.; FUJIOGI, M.; KOUTSOGIANNAKI, S. COVID-19 pathophysiology: a review. Clinical Immunology, [S.L.], v. 215, p. 108427, jun. 2020.

Recebido: 11.08.2021

Aceito: 30.10 .2021

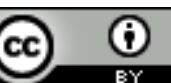

This work is licensed under a Creative Commons Attribution 4.0 International License.

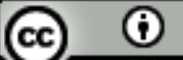

Este trabalho está licenciado com uma Licença Creative Commons - Atribuição 4.0

Internacional.

Revista Extensão \& Cidadania, v. 9, n. 16, p. 178-188, jul./dez. 2021.

ISSN 2319-0566 DOI: 10.22481/recuesb.v9i16.9271 